\title{
Seis años de experiencia en cirugía digestiva asistida por robot
}

\author{
James Hamilton S. ${ }^{1}$, Camila Onetto C. ${ }^{2}$, Pablo Marín P. ${ }^{1}$ y Omar Orellana E. ${ }^{3}$
}

\section{6 years of experience with robotic assisted gastrointestinal surgery}

Background: Since its approval, robotic surgery has been growing as a useful, promising and probably superior technique for many surgeries. The aim of this study is to expose and evaluate the results of a 6-year experience, of a single surgeon, with robotic assisted surgery, used for different gastrointestinal procedures throughout this years. Study Design: We conducted a retrospective revision of all consecutive robotic assisted gastrointestinal cases performed by a single gastrointestinal laparoscopic surgeon of the Bariatric Surgery Department and Robotics Surgery Department of Clínica Santa María of Santiago, Chile, between September 2011 and May 2017. Results: A total of 290 patients underwent robotic gastrointestinal surgery during 6 years. All cases were performed by the same gastrointestinal surgeon. Primary procedures performed were totally hand sewn Roux-en-Y gastric bypass, sleeve gastrectomy, revisional bariatric and non-bariatric surgery, Nissen fundoplication, single port cholecystectomy, Heller myotomy with Dor fundoplication, hernia repairs, and other exceptional cases. A total of 512 procedures were performed in 290 patients. The majority of secondary procedures were cholecystectomies, hiatal hernia repairs and adhesiolysis. Average length of hospital stay was 3 days. Postoperative complications were observed in 27 (9.3\%) patients. According to Clavien Dindo classification of surgical complications, 4 (1.4\%) complications were Clavien I, 5 (1.7\%) Clavien II, 18 (6.2\%) Clavien III, 0 Clavien IV, 0 Clavien V. There were no deaths in this group. Mean operative time was 76 minutes. Mean surgical time was 64 minutes. Conclusion: Robot assisted upper gastrointestinal surgery is a reliable and effective technique, that can be used in many diseases.

Key words: robotic assisted gastrointestinal surgery; robotic surgery, robotic gastrointestinal surgery.

\section{Resumen}

Introducción: Desde su aprobación, la cirugía robótica ha crecido como una técnica útil, prometedora y probablemente superior en muchas cirugías. El objetivo de este estudio es exponer y evaluar los resultados de 6 años de experiencia, en cirugía digestiva asistida por robot, utilizada en diferentes procedimientos gastrointestinales, a lo largo de estos años. Material y Método: Realizamos una revisión retrospectiva de todos los pacientes consecutivos sometidos a cirugía asistida por robot, realizada por un cirujano digestivo del Departamento de Cirugía Bariátrica y Departamento de Cirugía Robótica de la Clínica Santa María, de Santiago de Chile, entre septiembre de 2011 y mayo de 2017. Resultados: Un total de 290 pacientes fueron sometidos a cirugía digestiva asistida por robot durante 6 años. Todos los pacientes fueron operados por el mismo cirujano digestivo. Los procedimientos primarios realizados fueron bypass gástrico en Y-Roux con anastomosis manual, gastrectomía en manga, cirugía revisional bariátrica y no bariátrica, fundoplicatura de Nissen, colecistectomía con puerto único, miotomía de Heller con fundoplicatura de Dor, hernioplastías, y otros casos excepcionales. La mayoría de los procedimientos secundarios fueron colecistectomías, hernioplastías hiatales y adherenciolisis. La estadía hospitalaria promedio fue de 3 días. Se observaron complicaciones postoperatorias en $27(9,3 \%)$ pacientes. De acuerdo a la clasificación de Clavien Dindo, 4 (1,4\%) complicaciones fueron Clavien I, 5 (1,7\%) Clavien II, 18 (6,2\%) Clavien III, 0 Clavien IV, 0 Clavien V. No hubo mortalidad en este grupo. El tiempo total promedio de los procedimientos fue de $76 \mathrm{~min}$. El tiempo quirúrgico promedio fue de $64 \mathrm{~min}$. Conclusión: La cirugía digestiva asistida por robot es una técnica confiable y segura, que se puede utilizar para muchas enfermedades.

Palabras clave: cirugía digestiva asistida por robot; cirugía robótica, cirugía digestiva robótica.

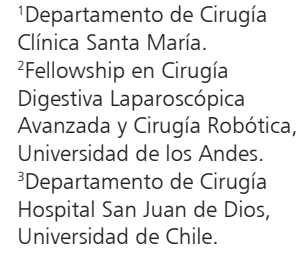

Recibido el 14 de agosto de 2017, aceptado para publicación el 8 de noviembre de 2017.

Correspondencia a: Camila Onetto camilaonetto@gmail.com 


\section{Introducción}

Desde su aprobación por la FDA en el año 2005, la cirugía robótica se ha desarrollado como una técnica útil, prometedora y probablemente superior en muchos procedimientos quirúrgicos ${ }^{1}$.

En múltiples departamentos de urología, la introducción y difusión de la cirugía robótica se encuentra en fases avanzadas, con un $85 \%$ de las prostatectomías radicales realizadas con esta técnica. En cirugía digestiva, esto no ha avanzado de forma tan rápida y efectiva, a pesar de que ha sido descrita para múltiples procedimientos realizados regularmente.

Como toda técnica, la cirugía laparoscópica tiene limitaciones, especialmente, en procedimientos de complejidad mayor. Estas limitaciones se relacionan con dificultades técnicas, instrumental sin extremos distales articulados, visión en dos dimensiones, la necesidad de un asistente para manipular la cámara, y una curva de aprendizaje larga para algunos de estos procedimientos. La cirugía robótica podría mejorar los resultados, mediante el control de las limitaciones descritas previamente, por ejemplo, teniendo instrumental articulado, visión en tres dimensiones, controlada por el cirujano ${ }^{2,3}$.

En los últimos años, la cirugía digestiva robótica ha adquirido mayor protagonismo, representando, una posible evolución de la cirugía laparoscópica ${ }^{4,5}$.

El objetivo de este estudio es describir y evaluar los resultados de 6 años de experiencia, en cirugía digestiva asistida por robot, utilizada para diferentes procedimientos gastrointestinales a través de los años en una institución médica.

\section{Material y Métodos}

Se realizó una revisión retrospectiva de todos los casos de pacientes sometidos a cirugía digestiva asistida por robot por un cirujano digestivo del Departamento de Cirugía Bariátrica y Departamento de Cirugía Robótica de la Clínica Santa María, en Santiago de Chile, entre septiembre del año 2011 y mayo del año 2017. Todos los procedimientos fueron realizados utilizando el mismo robot (DaVinci SI).

290 pacientes fueron sometidos a cirugía digestiva asistida por robot, en los cuales se realizaron un total de 512 procedimientos. Se registraron las características demográficas de los pacientes, sus comorbilidades, indicaciones de cirugía, número de procedimientos en cada cirugía, pérdidas estimadas de sangre, tiempo de estadía hospitalaria, compli- caciones postoperatorias y morbilidad a corto plazo (<30 días).

\section{Resultados}

Un total de 290 pacientes fueron sometidos a cirugía digestiva asistida por robot durante los 6 años, entre septiembre del año 2011 y mayo del año 2017. Las características demográficas se presentan en la Tabla 1.

Todos los procedimientos fueron realizados por el mismo cirujano digestivo. Un importante número de indicaciones fueron abordadas utilizando la técnica quirúrgica asistida por robot. Dentro de los procedimientos primarios, se realizaron bypass gástrico en Y-Roux con anastomosis manuales, gastrectomías en mangas, cirugía revisional bariátrica y no bariátrica, fundoplicatura de Nissen, colecistectomía con puerto único, miotomía de Heller y fundoplicatura de Dor, hernioplastías inguinales, y otros casos excepcionales, descritos en la Tabla 2.

En 8 pacientes, se realizaron 4 procedimientos durante la misma cirugía, en 50 pacientes, 3 procedimientos, en 164 pacientes, 2 procedimientos. Se realizaron un total de 512 procedimientos en los 290 pacientes. La mayoría de los procedimientos secundarios fueron colecistectomías, hernioplastías hiatales y adherenciolisis.

Tabla 1. Características demográficas de los pacientes

\begin{tabular}{|lr|}
\hline n de pacientes & 290 \\
Género & \\
- Masculino & $130(45 \%)$ \\
- Femenino & $160(55 \%)$ \\
Edad promedio (años) & 48 \\
Comorbilidades & \\
- Diabetes mellitus & $58(20 \%)$ \\
- Prediabetes & $62(21 \%)$ \\
- SAHOS & $33(11 \%)$ \\
- Hipertensión arterial & $77(27 \%)$ \\
- Patología vesicular & $47(16 \%)$ \\
- Hígado graso & $86(30 \%)$ \\
- Dislipidemia & $97(33 \%)$ \\
- Hernia hiatal & $74(26 \%)$ \\
- ERGE* & $124(43 \%)$ \\
IMC Promedio $\left(\mathrm{kg} / \mathrm{m}^{2}\right)^{*}$ & 31,69 \\
\hline
\end{tabular}

*Enfermedad por reflujo gastroesofágico. *Índice de masa corporal. 
Tabla 2. Intervenciones quirúrgicas primarias

\begin{tabular}{|ll|}
\hline Intervención primaria & $\begin{array}{c}\text { n de } \\
\text { pacientes }\end{array}$ \\
Bypass gástrico y-Roux & $88(30 \%)$ \\
Cirugía revisional & $63(22 \%)$ \\
- Remodelamiento manga & 11 \\
- Bypass gástrico y roux & 35 \\
- Extracción banda gástrica & 2 \\
- Extracción banda gástrica y gastrectomía & \\
en manga & 1 \\
- Banda gástrica a bypass gástrico y roux & 5 \\
- Gastrectomia total & 3 \\
- Gastro entero anastomosis & 1 \\
- Reparacion hiatal & 3 \\
- Resección fístula gastro gástrica & 1 \\
Gastrectomía en manga & $24(8 \%)$ \\
Fundoplicatura Nissen y hernioplastía hiatal & $47(16 \%)$ \\
Colecistectomía puerto único & $31(11 \%)$ \\
Miotomía Heller y fundoplicatura de Dor & $16(6 \%)$ \\
Otros & $21(7 \%)$ \\
- Pancreatectomía distal & 1 \\
- Hernioplastía inguinal, umbilical, & 12 \\
- Epigástrica, incisional & 1 \\
- Esplenectomía & 1 \\
- Coledoco duodeno anastomosis & 1 \\
- Lesección sarcoma retroperitoneal & 2 \\
- Gastrectomía total por cáncer gástrico & 1 \\
- Reparación hernia morgagni & 1 \\
- Divertículo esofágico & 1 \\
\hline
\end{tabular}

El tiempo promedio de estadía hospitalaria fue de 3 días. Se observaron complicaciones postoperatorias en $27(9,3 \%)$ de los pacientes. De acuerdo a la clasificación de Clavien Dindo de complicaciones quirúrgicas, $4(1,4 \%)$ complicaciones fueron Clavien I, 5 (1,7\%) Clavien II, 18 (6,2\%) Clavien III, 0 Clavien IV, 0 Clavien V. No hubo mortalidad en este grupo de pacientes. Las complicaciones postoperatorias precoces $(<30$ días $)$, observadas en cada subgrupo de pacientes, están descritas en la Tabla 3.

El tiempo operatorio total promedio fue de $76 \mathrm{~min}$. El tiempo quirúrgico promedio fue de $64 \mathrm{~min}$. Los tiempos de montaje, cirugía, complicaciones y estadía hospitalaria para cada grupo de procedimientos se resumen en la Tabla 4.

\section{Discusión}

Algunas de las ventajas extraordinarias de la cirugía robótica son la visión en tres dimensiones, el aumento de los grados de libertad y la disponibilidad de instrumental articulado. Estas ventajas pueden mejorar la precisión en la disección, reducir la fatiga en los cirujanos, mejorar los resultados en el cierre de las anastomosis intestinales, y podrían también, reducir las complicaciones relacionadas con filtraciones, estenosis y sangrado ${ }^{6}$.

Actualmente, una de las desventajas asociadas a la cirugía asistida por robot, es el costo adicional

Tabla 3. Complicaciones postoperatorias precoces

\begin{tabular}{|c|c|c|c|c|c|c|c|}
\hline & BGYR* & $\begin{array}{l}\text { Cirugía } \\
\text { Revisional }\end{array}$ & $\begin{array}{l}\text { Gastrectomía } \\
\text { en Manga }\end{array}$ & $\begin{array}{c}\text { Fundoplicatura Nissen y } \\
\text { Hernioplastía Hiatal }\end{array}$ & $\begin{array}{l}\text { Colecistectomía } \\
\text { por puerto único }\end{array}$ & $\begin{array}{l}\text { Miotomía } \\
\text { Heller }\end{array}$ & Otros \\
\hline Complicaciones (\%) & $11(12,5)$ & $4(6,4)$ & $2(8,3)$ & $2(4,3)$ & $1(3,2)$ & $2(12,5)$ & $5(23,8)$ \\
\hline Clavien Dindo I & 0 & 0 & 0 & 0 & 1 & 0 & 3 \\
\hline Clavien Dindo II & 2 & 1 & 1 & 1 & 0 & 0 & 0 \\
\hline Clavien Dindo III & 9 & 3 & 1 & 1 & 0 & 2 & 2 \\
\hline Clavien Dindo IV & 0 & 0 & 0 & 0 & 0 & 0 & 0 \\
\hline
\end{tabular}

Tabla 4. Características quirúrgicas

\begin{tabular}{|c|c|c|c|c|c|c|c|}
\hline & BGYR* & $\begin{array}{c}\text { Cirugía } \\
\text { Revisional }\end{array}$ & $\begin{array}{l}\text { Gastrectomía } \\
\text { en Manga }\end{array}$ & $\begin{array}{l}\text { Fundoplicatura Nissen } \\
\text { y Hernioplastía Hiatal }\end{array}$ & $\begin{array}{l}\text { Colecistectomía } \\
\text { por puerto único }\end{array}$ & $\begin{array}{l}\text { Miotomía } \\
\text { Heller }\end{array}$ & Otros \\
\hline $\mathrm{N}^{\mathrm{o}}$ Pacientes & 88 & 63 & 24 & 47 & 31 & 16 & 21 \\
\hline Estadía hospitalaria (días) & 3,8 & 3,6 & 2,3 & 1,7 & 1,2 & 5,1 & 3 \\
\hline Tiempo de Docking (min) & 4,3 & 5,1 & 4,6 & 5,2 & 8,1 & 5,4 & 4,7 \\
\hline Tiempo quirúrgico (min) & 90,7 & 101,7 & 34,3 & 82,5 & 24,9 & 91,7 & 102,4 \\
\hline
\end{tabular}

*Bypass gástrico en Y-Roux. 
que representa para los pacientes. En nuestra institución, el costo asociado a la cirugía asistida por robot, en comparación a la misma cirugía realizada por laparoscopia, tiene un valor adicional fijo, de $\$ 990.000$ (U\$1.500), asociado a los insumos utilizados en el procedimiento asistido por robot.

Se observaron complicaciones en el 9,7\% de los pacientes. De ellos, el 6,6\% requirió una intervención adicional para resolver dicha complicación. No hubo fallecidos dentro del grupo de estudio. Como resultado global, las complicaciones asociadas a la cirugía asistida por robot, fueron relativamente similares a la cirugía digestiva alta laparoscópica.

Los pacientes sometidos a bypass gástrico en Y-Roux, con anastomosis manual, presentaron una estadía hospitalaria corta, con un tiempo quirúrgico aceptable, observando complicaciones en el 12,5\% de ellos. De los 11 pacientes en los que se observaron complicaciones, 3 pacientes presentaron el diagnóstico de obstrucción intestinal, todos requirieron una exploración quirúrgica y resección del remanente gástrico, 2 de ellos con perforación de éste, secundario a la obstrucción. Cinco pacientes presentaron episodios de hemorragia digestiva alta, ninguno requirió una intervención quirúrgica adicional. De los pacientes restantes donde se observaron complicaciones, una presentó un tromboembolismo pulmonar agudo segmentario y subsegmentario del lóbulo inferior derecho, asociado a infarto pulmonar, otra un derrame pleural leve de manejo conservador y ambulatorio y finalmente, un paciente presentó una úlcera de la boca de la gastroyeyuno anastomosis, sin sangrado asociado a ésta. De los pacientes que requirieron una reintervención quirúrgica, los 3 están dentro de los primeros 45 pacientes sometidos a este procedimiento utilizando la técnica asistida por robot.

En otras series, se han reportado complicaciones menores al 10\% de los pacientes. La curva de aprendizaje para el bypass gástrico en Y-Roux robótico, se ha estimado en 40 pacientes. Esto podría tener una importante influencia en el porcentaje mayor de complicaciones observado en este grupo de pacientes, asociado al número total de pacientes de nuestra serie, que podría estar influyendo en el porcentaje elevado de complicaciones observado. Todas las complicaciones que requirieron procedimientos endoscópicos o reoperaciones, fueron en pacientes que se encuentran dentro de los primeros 45 que se operaron utilizando esta técnica ${ }^{2}$.

Los procedimientos revisionales, generalmente se asocian a mayores porcentajes de morbilidad, en comparación con los procedimientos primarios ${ }^{7}$. La cirugía revisional, por ejemplo, extracción de
BGA y conversión a BGYR, presenta incidencia de complicaciones postoperatorias alta, incluso del $10 \%$ o más ${ }^{8}$.

En esta revisión, se encontró una baja morbilidad asociada a la cirugía revisional asistida por robot $(6,35 \%)$, y no hubo mortalidad. Solamente 2 pacientes requirieron una nueva intervención quirúrgica, uno por biliperitoneo secundario a un conducto de Luchska inadvertido durante la colecistectomía que fue realizada durante la misma cirugía, y otro por hemoperitoneo en un bypass gástrico Y-Roux revisional. Un tercer paciente presentó una hemorragia digestiva alta autolimitada, que no requirió intervenciones adicionales.

A pesar de ser procedimientos que generalmente requieren de un mayor tiempo quirúrgico, principalmente por las adherencias, en esta serie, el tiempo quirúrgico promedio fue menor a $2 \mathrm{~h}$ incluyendo pacientes con cirugías de mayor complejidad una gastrectomía total. Las gastrectomías en manga fueron realizadas como parte de la curva de aprendizaje. Muchos autores sugieren que, una buena forma de iniciar la experiencia en cirugía robótica, es con procedimientos como la gastrectomía en manga, y así, ir escalando a procedimientos de mayor complejidad como el bypass gástrico en Y-Roux. El porcentaje de complicaciones en estos pacientes fue de $8,3 \%$, lo que es alto, considerando los bajos porcentajes de complicaciones en la cirugía bariátrica laparoscópica ${ }^{9,10}$. De los 2 pacientes que presentaron complicaciones, una paciente evolucionó con una colección intraabdominal que se resolvió mediante drenaje percutáneo y tratamiento antibiótico, y el otro paciente presentó una neumonía que se manejó con antibióticos de forma ambulatoria. Una posible explicación para este porcentaje, es que este grupo de pacientes fue parte de la experiencia inicial en cirugía asistida por robot, y la cantidad total de pacientes, operados utilizando esta técnica, es baja.

La fundoplicatura laparoscópica se ha convertido en el procedimiento quirúrgico estándar para la enfermedad por reflujo gastroesofágico. Estudios previos han demostrado que la fundoplicatura asistida por robot es factible, segura y efectiva, sin embargo, no se ha demostrado aún, que sea una técnica superior a la cirugía laparoscópica ${ }^{2}$. Lo observado en este grupo de pacientes es que, la fundoplicatura de Nissen asistida por robot es un procedimiento seguro y efectivo, con un porcentaje de complicaciones similar a la técnica laparoscópica, corta estadía hospitalaria, menor a 2 días, y tiempos quirúrgicos aceptables. Hasta ahora, la colecistectomía asistida por robot impresiona ser segura y efectiva, sin embargo, no existen evidencias de ventajas signifi- 
cativas por sobre la laparoscopia estándar². En esta serie de pacientes, se observó que la colecistectomía asistida por robot con puerto único, es un procedimiento seguro, confiable y efectivo. Los pacientes presentaron tiempos quirúrgicos cortos, bajo porcentaje de complicaciones y estadía hospitalaria corta. A pesar de lo descrito, se requieren mayores estudios comparativos entre la técnica con puerto único laparoscópica y la asistida por robot para demostrar beneficios en este tipo de cirugía.

En el tratamiento quirúrgico laparoscópico de la acalasia, el porcentaje de perforación de estómago o esófago durante la cirugía está descrito en aproximadamente $11 \%$ de los pacientes. Estudios de miotomía de Heller asistida por robot han reportado $0 \%$ de perforaciones durante el procedimiento ${ }^{2}$. En esta serie de pacientes, 16 fueron sometidos a una miotomía de Heller y fundoplicatura de Dor asistida por robot. Del total, 2 pacientes presentaron una perforación esofágica $(12,5 \%)$. Este porcentaje podría reducirse en la medida que aumente el número de pacientes operados utilizando esta técnica, ya que, no se han observado perforaciones en los últimos 6 pacientes de este grupo.

Procedimientos de mayor complejidad como gastrectomía total con linfadenectomía por cáncer gástrico o pancreatectomías distales, han sido descritas con técnica asistida por robot, observando buenos resultados hasta ahora. Sin embargo, se requiere de mayor experiencia para demostrar que esta técnica es mejor que la cirugía laparoscópica, considerando las ventajas mencionadas previamente ${ }^{11}$.

\section{Conclusión}

La cirugía digestiva alta asistida por robot es una técnica confiable y efectiva, que se puede utilizar para la resolución de múltiples enfermedades. Se requieren estudios adicionales para demostrar su posible superioridad en comparación con la técnica laparoscópica, especialmente, en procedimientos de mayor complejidad como el bypass gástrico en YRoux, la cirugía revisional, resección de neoplasias gastrointestinales, donde la disección, anastomosis y porcentaje de filtraciones, podrían tener mejores resultados con la cirugía robótica ${ }^{10}$.

\section{Responsabilidades éticas}

Protección de personas y animales. Los autores declaran que para esta investigación no se han realizado experimentos en seres humanos ni en animales.

Confidencialidad de los datos. Los autores declaran que en este artículo no aparecen datos de pacientes.

Derecho a la privacidad y consentimiento informado. Los autores declaran que en este artículo no aparecen datos de pacientes.

\section{Conflicto de intereses}

Los autores declaran no tener ningún conflicto de intereses.

\section{Bibliografía}

1. Garbura O, Tomulescu V, Blajut C, Popescu I. A 5-Year Perspective over Robotic General Surgery: Indications, Risk Factors and Learning Curves. Chirurgia 2013;108:599-610.

2. Brunaud L, Reibel N, Ayav A. Pancreatic, endocrine and bariatric surgery: The role of robot-assisted approaches. Journal of Visceral Surgery 2011;148:e47-e53.

3. Ayloo S, Roh Y, Choudhury N. Laparoscopic, hybrid, and totally robotic Roux-en-Y gastric bypass. J Robotic Surg. 2016;10:41-7.

4. Parisi A, Desiderio J, Trastulli S, Cirocchi R, Renzi C, Boselli C, et al. Robotic pylorus-preserving pancreaticoduodenectomy: Technical considerations. International Journal of Surgery 2015;21:59-63.

5. Kun L, Jianan Z, Jianxiong T, Jianxhong D, Xiaodong H, Pin Z. Robotic Versus Laparoscopic Bariatric Surgery: a Systematic Review and Meta-Analysis. Obes Surg. 2016;26:3031-44.

6. Suk Y, Hoon S, Joong D, Ho Kim H. Bariatric and revisional robotic surgery. Transl Gastrointest Cancer 2016;5:1-7.

7. Subhashini M, Nabajit C. Robotic revisional bariatric surgery: single-surgeon case series. Int J Med Robotics Comput Assist Surg. 2015;11:284-9.

8. Wezenbeek M, Oudheusden T, Zoete J, Smulders J, Nienhuijs S. Conversion to Gastric Bypass After Either Failed Gastric
Band or Failed Sleeve Gastrectomy. Obes Surg. 2017;27: 83-9.

9. Gadiot R, Biter L, van Mil S, Zengerink H, Apers J, Mannaerts G. Long-Term Results of Laparoscopic Sleeve Gastrectomy for Morbid Obesity: 5 to 8 -Year Results. Obes Surg. 2017;27:59-63.

10. Magouliotis D, Tasiopoulou V, Sioka E, Zacharoulis D. Robotic versus Laparoscopic Sleeve Gastrectomy for Morbid Obesity: a Systematic Review and Meta-analysis. Obes Surg. 2017;27:24553.

11. Koichi S, Masaya N, Kazuki I, Yoshinori I, Ichiro U. Minimally invasive surgery for upper gastrointestinal cancer: Our experience and review of the literature. World J Gastroenterol. 2016;22:4626-37. 